\title{
Bilateral total hip arthroplasty as a treatment for ollier's disease of the proximal femur: a case report
}

\begin{abstract}
Ollier's disease is very rare condition that is characterized by the presence of multiple cartilaginous tumors in the long tubular bones which can lead to severe deformities and pathological fractures at early age. More commonly the lesions have a unilateral distribution. We report a case of an adult patient with medical history of Ollier's disease involving both hips treated with a 2-stage bilateral total hip arthroplasty (THA) using a cement less ace tabular cup and a short cement less femoral stem, achieving excellent clinical and radiographic results.
\end{abstract}

Keywords: Ollier's disease, Enchondromatosis, Total hip arthroplasty
Volume 5 Issue 6 - 2016

\author{
Cusmà Gianluca,' Ciliberto Ricardo,' \\ Caldarella Emmanuelle,' Grappiolo Guido² \\ 'Department of mininvasive orthopaedic surgery, Casa di Cura \\ Citta di Pavia, Italy \\ ${ }^{2}$ Department of Orthopedics of the Hip and Prosthetic Surgery, \\ Istituto Clinico Humanitas, Italy
}

\begin{abstract}
Correspondence: Cusma Gianluca, Department of mininvasive orthopedic surgery, Casa di Cura Cittadi Pavia, Pavia, Italy, Tel 39 329 6577343,Email gcd@me.com
\end{abstract}

Received: September 06, 2016 | Published: September 16, 2016

\section{Introduction}

Enchondromas are common benign usually asymptomatic cartilage tumors, which develop in the metaphyses and may become incorporated into the diaphyses of long tubular bones, in close proximity to growth plate cartilage. ${ }^{1-2}$ They usually occur as a single lesion (solitary enchondroma). Occasionally, multiples enchondromas may appear and it is defined as a enchondromatosis. Which encompasses several subtypes among which the most common are Ollier's Disease and Maffucci's Syndrome. The estimated prevalence of enchondromatosis is $1 / 100.000 .^{3}$

Ollier's disease is defined by the presence of multiple enchondromas ( 3 or more), and characterized by an asymmetric distribution of cartilaginous lesions that can be extremely variable in terms of size, number, location, age of onset and diagnosis, and requirement for surgery. ${ }^{4}$ It also can be associated with pathological fractures. ${ }^{5}$

The diagnosis is usually based on clinical and radiological features. Radiographies typically show multiple, radiolucent, homogenous lesions with an oval or elongated shape and well defined slightly thickened bony margin. ${ }^{2}$ Biopsy does not play an important role in the diagnosis. Further diagnostic analysis such as CT scan, MRI, scintigraphy, ultrasonography might be helpful during the follow-up.

Herein, we report on a case of Ollier's disease of the proximal femurs treated with a THA with a 2,5-year follow-up. After extensive literature review we could not find previous reports regarding this rare clinical presentation.

\section{Case presentation}

A 37-years-old male patient, with Ollier's disease and a 10-year history of mild-moderate pain in both hips and thighs associated to weakness and limp. Pain was mild at rest and exacerbated by walking. There was no history of trauma, fever or night pain. After several consultations he came to our department for further examinations and treatment.

Physical examination showed a limb discrepancy of $4 \mathrm{~cm}$. The range of motion of the right hip was $60-20^{\circ}$ of flexion and extension with a moderate flexion contracture. The range of motion of the left hip was $60^{\circ}-10^{\circ}$ of flexion and extension. Right hip internal rotation was to $15^{\circ}$ and external rotation to $30^{\circ}$, while for the left hip the internal rotation was to $15^{\circ}$ and external rotation was to $35^{\circ}$. His right hip passive abduction was limited to $10^{\circ}$, and adduction was similarly limited to $15^{\circ}$. The left hip had a passive abduction to $25^{\circ}$ and an adduction limited to $10^{\circ}$. At the end of the clinical examination the Harris Hip Score was 62. Therefore, a varus deformity of the left knee and valgus deformity of right knee were evident. The radiological findings suggested of multiple enchondromatosis of the both proximal femurs that involved the epiphysis and metaphysis.

In November 2013 we decided to perform a THA in the left side using a 54-mm cement less acet abular cup and short cement less femoral stem (GTS, Biomet) through a posterolateral approach with the Femur First Technique. Intraoperatively, the hip was stable with a good range of motion and no subluxation or impingement. The patient began physiotherapy the next morning and 18 hours after the procedure he was able to bear partial weight with assistance. There were no subsequent complications and the patient was discharged home after 12 days, according to our protocols with proper hip precautions. At 6-month follow-up the patient was able to bear complete weight, without any symptoms and could perform the daily activities.

At 2-year follow-up, clinical and radiographic results were excellent, and the patient was satisfied. On the clinical exam of the left hip the range of motion achieved $110^{\circ}$ of flexion. Internal rotation was to $20^{\circ}$ and external rotation to $35^{\circ}$. His left hip passive abduction was to $35^{\circ}$, and adduction was up to $20^{\circ}$ with a Harris Hip Score up to 75 . Thus, at this time point, we decided to schedule and perform a second THA on the right hip using the same materials and technique, achieving a remarkable outcome without any complications. At 1 year follow up, the patient was satisfied and was able to walk without limp. The clinical exam was notoriously improved achieving a Harris Hip Score up to 93 [Figure 1-3].

\section{Discussion}

Ollier's disease is a very rare condition, which symptoms frequently develop during the first decade of life, unlike our case. As stated above, it almost exclusively affects the metaphysis of long bones and the small bones of the hands and feet. ${ }^{2}$ There are very few 
reports of single enchondromas of the hip in english literature, ${ }^{5-6}$ and only one case of multiple enchondromas located on the hip like our patient.

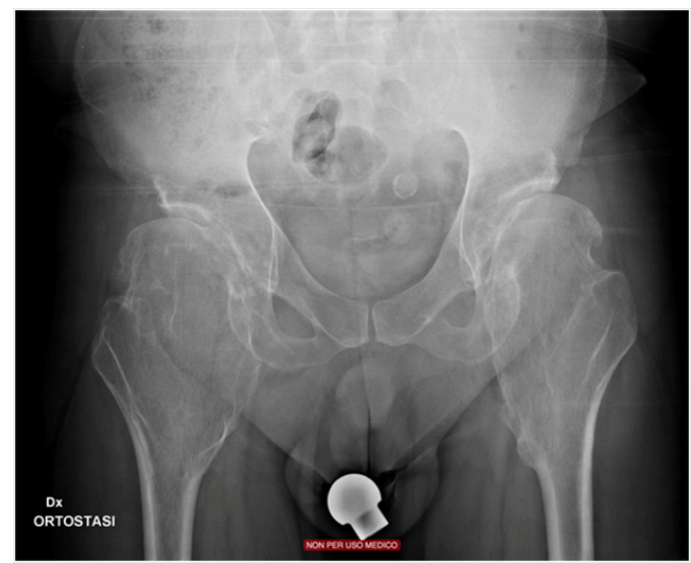

Figure I Preoperative radiography showing large enchondromas on both hips.

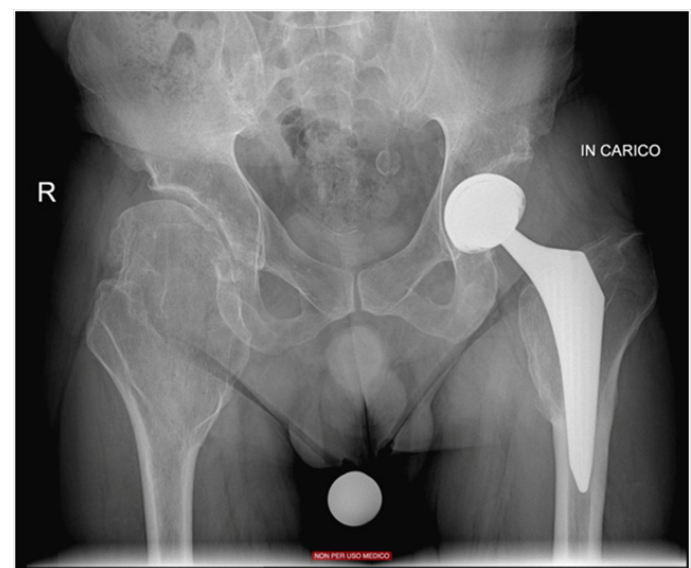

Figure 2 A I-year follow up x-ray shows the position of the femoral stem without any signs of loosening.

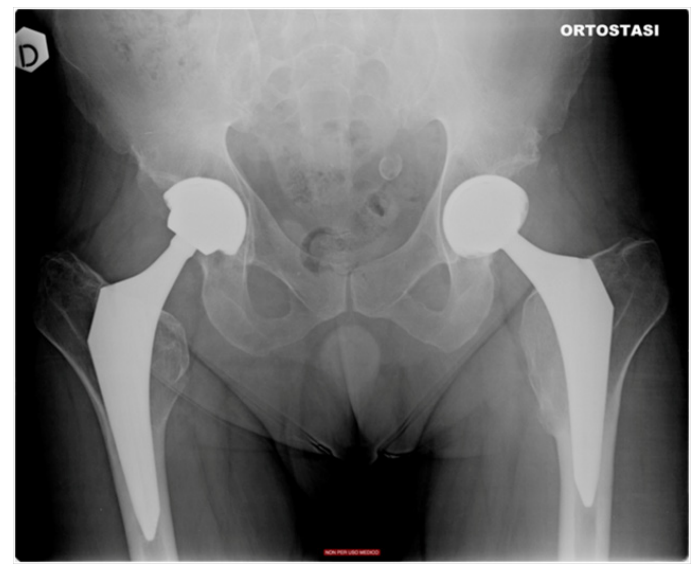

Figure 3 Absence of radio graphical modifications after 2.5 years of the first THA.

The patient was a 37-year-old male with an Ollier's disease that involved both hips. Clinical features were characteristic of this condition: limb inequality with asymmetric shortening of the right limb, loss of hip function and a flexion contracture. Additionally, a valgus and varus deviation of both knees was present. Treatment of Ollier disease is usually conservative and often the lesions are left untreated. Indications for surgery are very variable and predominantly depend on the presence of deformity, limb-length discrepancy, pathological fracture, and malignant transformation. According to Kumar et al. the goals of the treatment are achieving mechanical alignment and equivalent limb length for normal walking, and relieving pain from a pathological fracture. ${ }^{7}$

Due to the characteristics and localization of the enchondromas, there is not a standardized surgical treatment. ${ }^{7}$ In this particular case, considering the anatomic localization of the lesions in both proximal femurs, without involving the diaphysis, and the functional limitation of the hip joints, we proposed a bilateral THA performed in a twostage intervention. Given the lack of evidence in this matter, we first performed the left hip arthroplasty and, in the view of the remarkable results that were achieved at 1-year follow-up, we then proceeded to the second THA.

Ollier's disease might be a challenging condition to treat, because of the great variability not only in the clinical presentation, but also in the size, number and localization of the enchondromas. It is well known that surgical approach varies from case to case. Enchondromatosis with bilateral hip involvement is very unusual and, in our case, we considered THA was the best available therapeutic option, allowing us to improve substantially the joint anatomy and function. Thus, we believe total hip arthroplasty should be taken into consideration in similar cases.

\section{Acknowledgments}

None.

\section{Conflicts of interest}

None.

\section{References}

1. Maroteaux P, Merrer LM. Les maladies osseuses de l'enfant. MédecineSciences, Flammarion, Paris, France. 2002.

2. Whyte M. Acquired Disorders of Cartilage and Bone. American Society for Bone and Mineral Research, Washington DC, USA. 2003.

3. Spranger J, Kemperdieck H, Bakowski H, et al. Two peculiar types of enchondromatosis. PediatrRadiol. 1978;4(7):215-219.

4. Silve C, Juppner H. Ollier disease. Orphanet J Rare Dis. 20061(1):37.

5. Singh P, Kejariwal U, Chugh A. A rare occurrence of Enchondroma in neck of Femur in an adult female: A case report. $J$ Clin Diagn Res. 2015;9(12):RD01-RD03.

6. Mori S, Miyazaki N, Inoue YA. Case Report of the Solitary Enchondroma Originating From Neck of the Femur. Orthopedics \& Traumatology. 1986;35(2):377-379.

7. Kumar A, Jain VK, Bharadwaj M, et al. Ollierdisease: Pathogenesis, diagnosis, and management. Orthopedics. 2015;38(6):e497-e506. 